\title{
Live-Cell Epigenome Manipulation by Synthetic Histone Acylation Catalyst System
}

\author{
Yusuke Fujiwara, ${ }^{\dagger}$ Yuki Yamanashi, ${ }^{\dagger}$ Yuko Sato, ${ }^{\star}$ Tomoya Kujirai,${ }^{\perp}$ Hitoshi Kurumizaka,${ }^{\perp}$ \\ Hiroshi Kimura, ${ }^{\dagger}$ Kenzo Yamatsugu, ${ }^{*}{ }^{\dagger}$ Shigehiro A. Kawashima, ${ }^{* \dagger}$ \& Motomu Kanai ${ }^{*}, \dagger$ \\ ${ }^{\dagger}$ Graduate School of Pharmaceutical Sciences, The University of Tokyo, 7-3-1 Hongo, Bunkyo-ku, Tokyo 113-0033, Japan \\ ${ }^{\ddagger}$ Cell Biology Center, Institute of Innovative Research, Tokyo Institute of Technology, 4259 Nagatsuta, Midori-ku, Yoko- \\ hama, 226-8503, Japan \\ ${ }^{\perp}$ Institute for Quantitative Biosciences, The University of Tokyo, 1-1-1 Yayoi, Bunkyo-ku, Tokyo, 113-0032, Japan
}

\section{Supporting Information Placeholder}

\begin{abstract}
Chemical modifications of histones play a pivotal role in the epigenome and regulation of gene expression, and their abnormality is tightly linked to numerous disease states in humans. Therefore, chemical tools to manipulate epigenome hold promise for both therapy and the elucidation of epigenetic mechanisms. We previously developed the chemical catalyst LANA-DSH, which binds to nucleosomes via a LANA peptide ligand, and selectively acylates proximal histone H2BK120 to the catalyst moiety by activating acyl-CoAs. Thus far, however, histone acylation by a chemical catalyst system in living cells has not yet been demonstrated. Here, we report a chemical catalyst system, composed of a nucleosome-binding catalyst (PEG-LANA-DSH) and a cell-permeable thioester acyl donor (NAC-acyl), that can promote regioselective lysine acylation of histones in living cells. Whereas LANA-DSH is rapidly decomposed in cells, attachment of polyethylene glycol (PEG) to the LANA moiety can prevent this undesired degradation. Increasing the size of PEG conferred LANA with greater in-cell stability, but reduced catalytic activity, indicating that there is an optimum PEG length balancing stability and catalytic activity. The optimized PEG-LANA-DSH catalyst $\mathbf{1 1}$ efficiently promoted H2BK120 acetylation in living cells, which subsequently suppressed ubiquitination of H2BK120, a mark regulating various chromatin functions, such as transcription and DNA damage response. Thus, our chemical catalyst system will be useful as a unique tool to manipulate the epigenome for therapeutic purposes or further understanding epigenetic mechanisms.
\end{abstract}

The eukaryotic epigenome is defined by multiple chemical modifications of biomacromolecules, including DNA methylation and histone post-translational modifications (PTMs), and plays a pivotal role in regulating gene expressions. Dysregulation of the epigenome is tightly linked to numerous disorders in humans, such as type 2 diabetes, Alzheimer disease, and various types of cancer. ${ }^{1-3}$ Thus, new methods to manipulate the epigenome hold promise for both therapy and elucidation of epigenetic mechanisms. The levels of histone PTMs are regulated by the interplay of two groups of enzymes; writers which introduce PTMs (e.g. histone acetyltransferase, HAT) and erasers which remove them (e.g. histone deacetylase, HDAC). One approach to manipulate histone PTMs is use of small molecule inhibitors of these chromatin-modifying enzymes.
So far, several HDAC inhibitors have been approved for the treatment of cancer, and further candidates are under clinical evaluation. ${ }^{4}$

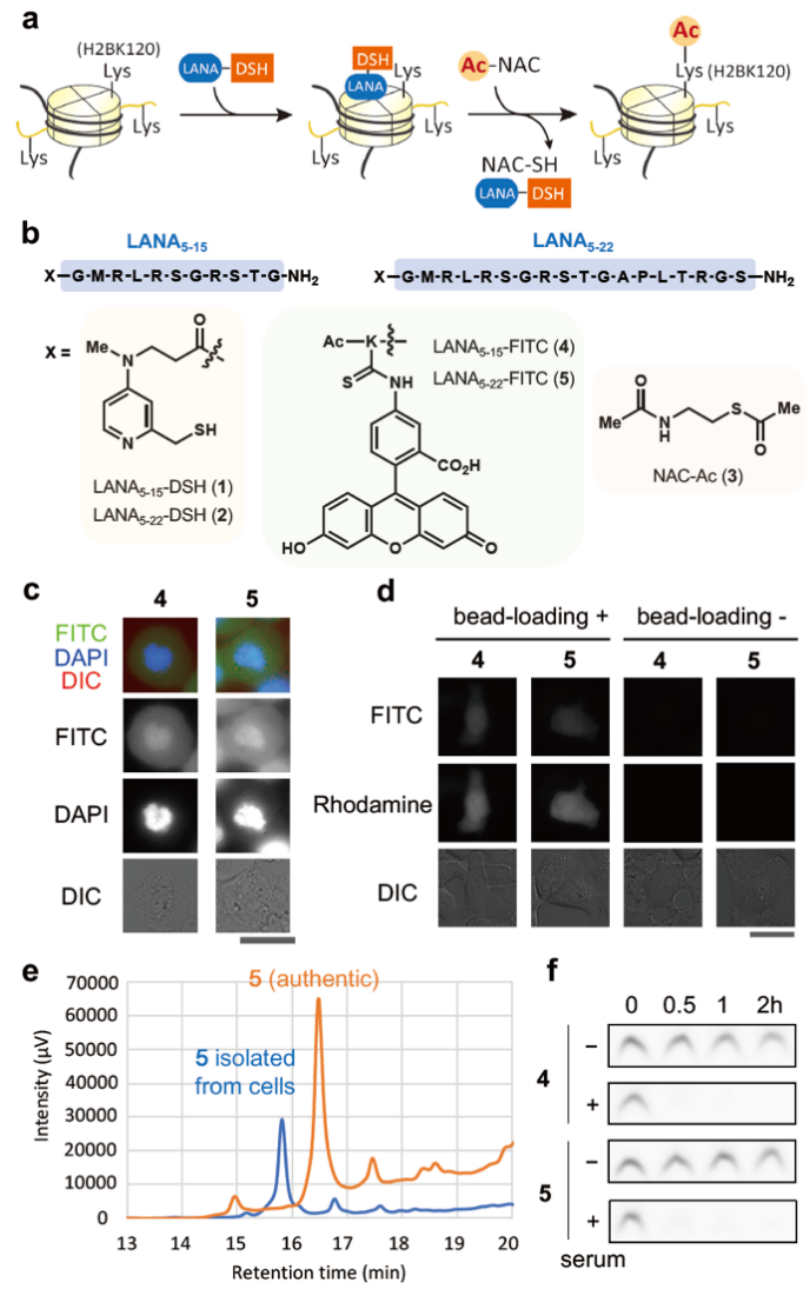

Figure 1. Histone-acylation catalyst LANA-DSH is unstable in living cells. (a) Schematics of site-selective histone acetylation by LANA-DSH. LANA-DSH binds to nucleosomes, activates the acetyl donor (NAC-Ac), and acetylates H2BK120. (b) Structures of 


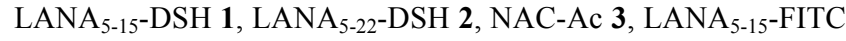

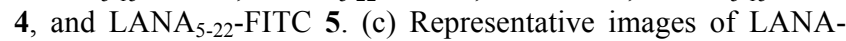
FITC (4 and 5) with DAPI and DIC in fixed HeLa S3 cells. Scale bar $=25 \mu \mathrm{m}$. (d) Representative images of 4 and 5 in living HeLa $\mathrm{S} 3$ cells. (Left) $100 \mu \mathrm{M}$ of LANA-FITC was introduced with Dextran-Rhodamine (loading control) by bead-loading. (Right) $50 \mu \mathrm{M}$ LANA-FITC was treated with cells for $30 \mathrm{~min}$. Scale bar $=25 \mu \mathrm{m}$. (e) HPLC traces of chart of 5 with fluorescence detection ( $\mathrm{ex}=494$ $\mathrm{nm}$, em $=518 \mathrm{~nm}$ ). Authentic peptide 5 (orange), and recovered peptide from the cells (blue). (f) Serum stability assay of $\mathbf{4}$ and $\mathbf{5}$. Each peptide was incubated with or without $25 \%$ human serumcontaining medium at $37^{\circ} \mathrm{C}$ for indicated time.

In recent years, new chemical biology approaches that introduce histone PTMs entirely through artificial means without reliance on chromatin-modifying enzymes have emerged. ${ }^{5}$ These include protein semi-synthesis using native chemical ligation, ${ }^{6}$ ribosome-mediated protein synthesis using an expanded genetic code, ${ }^{7}$ chemistry-coupled posttranslational mutagenesis, ${ }^{8-9}$ and synthetic histone acylation by chemical catalysis. ${ }^{10-11}$ Among these, the chemical catalysis approach is unique in that it does not require any genetic manipulations, and thus has great potential for therapeutic use. We previously developed the synthetic histone acylation catalyst LANA-DSH, which binds to nucleosomes via a LANA peptide ligand, and demonstrated in test tubes that LANA-DSH selectively acylates lysine 120 of histone H2B (H2BK120), a lysine residue proximal to the catalytically-active DSH moiety (Figure 1a). ${ }^{10}$ Thus far, however, in-cell histone acylation by LANA-DSH has not yet been demonstrated. Here we report a new catalyst system comprising PEG-LANA-DSH and NAC-Ac, enabling selective H2BK120 acetylation in living cells.
We first examined whether LANA-DSH can be applied for in-cell histone acylation. We used two types of LANA-DSH catalysts, LANA $_{5-15}$-DSH 1 and LANA $_{5-22}$-DSH 2, and NAC-Ac 3 as a cell-permeable acetyl donor (Figure $1 \mathrm{~b}$ ). ${ }^{12}$ As reported previously, ${ }^{10}$ these catalysts efficiently promoted acetylation of H2BK120 in recombinant nucleosomes (Figure S1). However, we could not detect H2BK120 acetylation by the catalyst system in living cells. Since LANA is composed of natural amino acids, we hypothesized that LANA-DSH catalysts were rapidly decomposed in living cells. To examine in-cell stability of LANA, we synthesized LANA $_{5-15}$-FITC 4 and LANA ${ }_{5-22}$-FITC 5 (Figure 1b). Using fixed mitotic cells, we confirmed that both compounds have affinity to chromatin (Figure 1c). However, when these compounds were introduced into living cells, they did not show any chromatin localization (Figure 1d). We analyzed 5 recovered from living cells by HPLC and found that the compound was almost completely decomposed (Figure 1e). Further, both $\mathbf{4}$ and $\mathbf{5}$ were immediately decomposed in the presence of human serum in vitro (Figure 1f). These data indicate that LANA-DSH catalysts are decomposed by proteases in living cells.

Conjugation with poly(ethylene glycol) [PEG] moieties (i.e., pegylation) has been used to improve the pharmacokinetic and pharmacodynamic properties of peptides and proteins. ${ }^{13}$ We expected that conjugation of a PEG moiety with a proper size to LANA should increase stability towards proteolysis. We thus synthesized LANA $_{5-22}$-FITC conjugated with three PEG chains, whose molecular weight was ca. 150 ( $\mathrm{PEG}_{150}$-LANA-FITC 6), 550

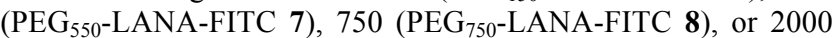
( PEG $_{2000}$-LANA-FITC 9) (Figure 2a). As a negative control, we also synthesized $\mathrm{PEG}_{0}$-LANA-FITC 10 without the PEG chains (Figure 2a). We compared stability of these compounds in the presence of human serum. While $\mathbf{1 0}$ and $\mathbf{6}$ were decomposed within 30 $\min , \mathbf{7}, \mathbf{8}$, and $\mathbf{9}$ were much more stable (Figure 2b). a
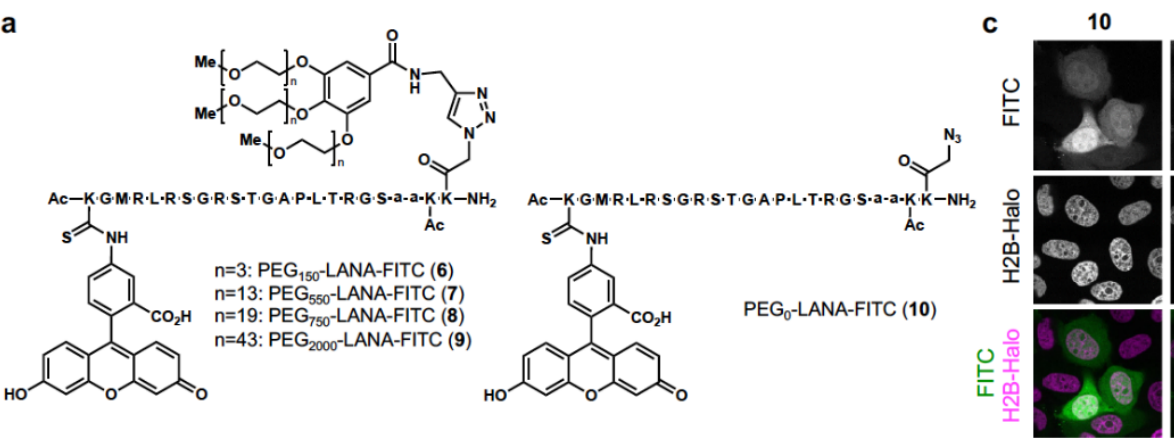
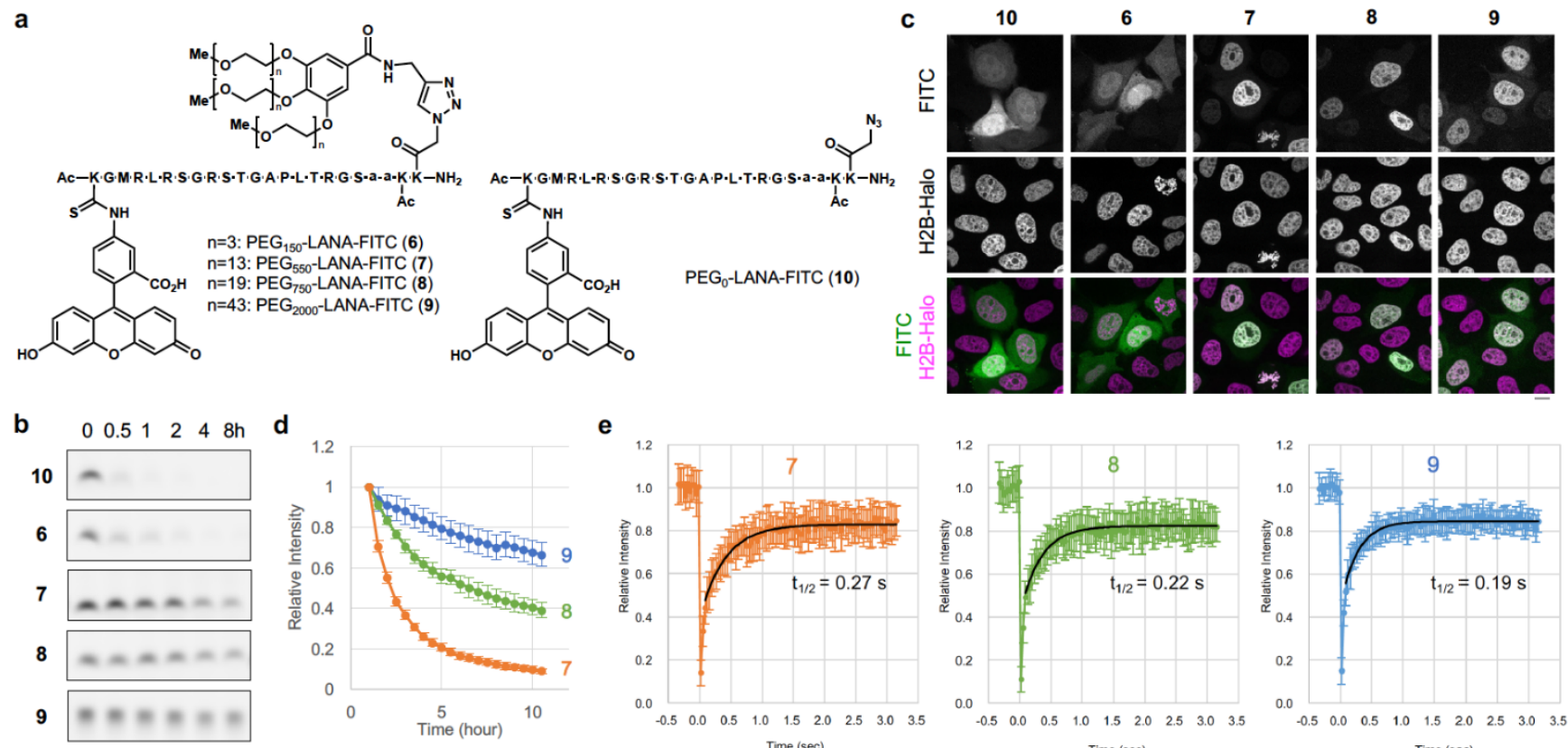

e
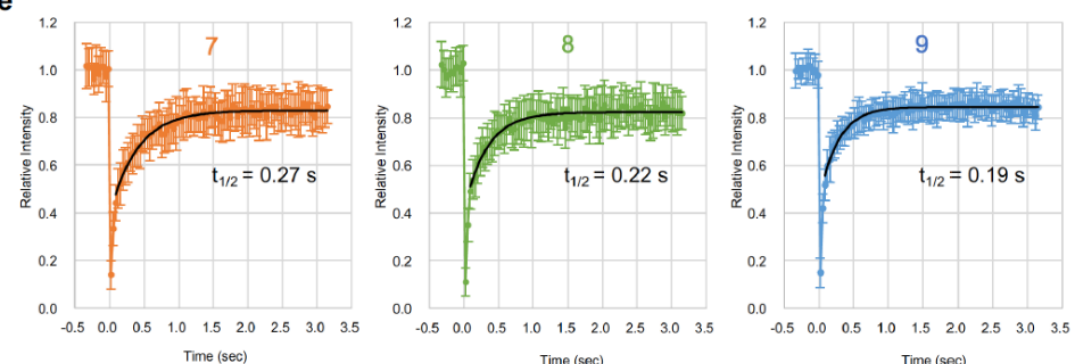

Figure 2. Conjugation of PEG stabilizes LANA in living cells. (a) Structures of PEG-LANA-FITC (6-10). (b) Serum stability assay of 610. Each peptide was incubated with $25 \%$ human serum-containing medium at $37{ }^{\circ} \mathrm{C}$ for indicated times. (c) Representative images of PEGLANA-FITC 6-10 in living cells. HeLa cells expressing H2B-HaloTag were stained with HaloTag TMR Ligand and loaded with PEGLANA-FITC. Scale bar $=10 \mu \mathrm{m}$. (d) Stability of 7-9 in living HeLa cells. Fluorescence images of PEG-LANA-FITC-loaded cells were acquired every $30 \mathrm{~min}$ and nuclear intensities were measured. Graphs represent relative fluorescence intensities (averages with standard deviations; $\mathrm{n}=12$ each). (e) Photobleaching was performed using HeLa cells loaded with 7-9. Average intensities with standard deviations $(\mathrm{n}=20)$ are shown with curves after fitting (black lines). Half-time recoveries are also indicated. 
We next examined the stability of the compounds in living cells. PEG-LANA-FITC compounds 6-10 were loaded into HeLa cells expressing H2B-HaloTag, and their localization were analyzed by fluorescent microscopy. If the compounds are stable in living cells, they bind with nucleosomes via the LANA moiety and localize to the chromatin regions, which are visualized by H2BHaloTag signals. Consistent with the serum stability assay, $\mathbf{1 0}$ and 6 did not localize on chromatin, indicating that these two compounds were immediately decomposed in living cells (Figure 2c). In contrast, the signals of 7,8 , and 9 colocalized with that of H2BHaloTag, indicating that these compounds are sufficiently stable in living cells and retain binding affinity of the LANA moiety to chromatin histones (Figure 2c). Time-lapse imaging showed that attachment of longer PEG chains conferred greater in-cell stability to LANA-FITC (Figure 2d). The approximate half-lives of 7, 8, and 9 were calculated to be $1.2 \mathrm{~h}, 5.3 \mathrm{~h}$, and $20 \mathrm{~h}$, respectively. We further examined residence times of the compounds on chromatin in living cells by fluorescence recovery after photobleaching (FRAP). In cells loaded with PEG-LANA-FITC, a small spot was photobleached and the rate of fluorescence recovery was measured (Figure $2 \mathrm{e}$ ). The recovery $\mathrm{t}_{1 / 2}$ corresponding to the residence time of 7 was $0.27 \mathrm{~s}$, indicating that 7 bound with chromatin transiently. Residence time of $8\left(t_{1 / 2}=0.22 \mathrm{~s}\right)$ or $9\left(t_{1 / 2}=0.19 \mathrm{~s}\right)$ was shorter than that of 7, suggesting that conjugation with longer PEG may reduce affinity of PEG-LANA-FITC with chromatin. Consistently, electrophoretic mobility shift assay of $\mathbf{7 - 1 0}$ showed that the conjugation with longer PEG chains weakened the binding to recombinant nucleosomes (Figure S2). Judging from the results of the FRAP experiments with 7, we estimated the dissociation constant $\left(K_{\mathrm{d}}\right)$ of 7 in living cells to be $\sim 100 \mu \mathrm{M}$ (Figure S3, see Supporting Information). This value was ca. 60 times higher than that in test tubes, suggesting that in-cell reaction may require higher concentrations of the catalysts than the reactions in test tubes. Taken together, these data demonstrate that conjugation of a longer PEG chains with the LANA ligand is advantageous for in-cell stability, but disadvantageous for LANA-mediated nucleosome binding.

We then synthesized chemical catalysts conjugated with PEG-LANA ligands (Figure 3a). As the catalyst moiety, we used DSSMe, a pro-drugged DSH catalyst, ${ }^{12}$ which is reduced to the active DSH form by biocompatible reductants, such as glutathione (GSH) or tris(2-carboxyethyl)phosphine (TCEP), prior to the reaction. The acetylation activity of the three catalysts $\left(\mathrm{PEG}_{550}-\mathrm{LANA}-\right.$ DSSMe 11, PEG $_{750}$-LANA-DSSMe 12, and PEG $_{2000}$-LANADSSMe 13) was first examined in test tubes containing recombinant nucleosomes as substrates. All the catalysts promoted acetylation of H2BK120 in recombinant nucleosomes, although the activity of PEG-LANA-DSH 11-13 was slightly weaker than that of LANA-DSH 1 and $\mathbf{2}$ (Figure 3b). Next, we addressed whether PEG-LANA-DSH catalysts can promote H2BK120 acetylation in living cells. The PEG-LANA-DSSMe catalysts were reduced by TCEP and loaded into HeLa S3 cells with rhodamine-labeled dextran as a loading control and L-buthionine-sulfoximine (BSO) pretreatment for reducing concentration of glutathione. After cells were treated with acetyl donor 3 for $8 \mathrm{~h}$ at $37^{\circ} \mathrm{C}$, cells with different levels of rhodamine signals (high, mid, or low) were sorted by flow cytometry (Figure 3c). Experiments using 7 suggested that the "high" fraction may contain $\sim 3.2 \times 10^{-16} \mathrm{~mol} 7$ in a cell, which corresponds to $427 \mu \mathrm{M}$ effective concentration in nuclei provided that all the catalysts loaded into the cells existed inside the nuclei, while the "mid" and "low" fractions may contain $0.67 \times 10^{-16}$ and 0.054 $\times 10^{-16} \mathrm{~mol} 7 /$ cell compounds, respectively (Figure S4). Comparing the yield of H2BK120 acetylation promoted by three PEG-LANADSSMe catalysts 11-13 in the "mid" cell fraction, we determined $\mathbf{1 1}$ to be the optimum chemical catalyst for in-cell reaction (Figure $3 d)$. a
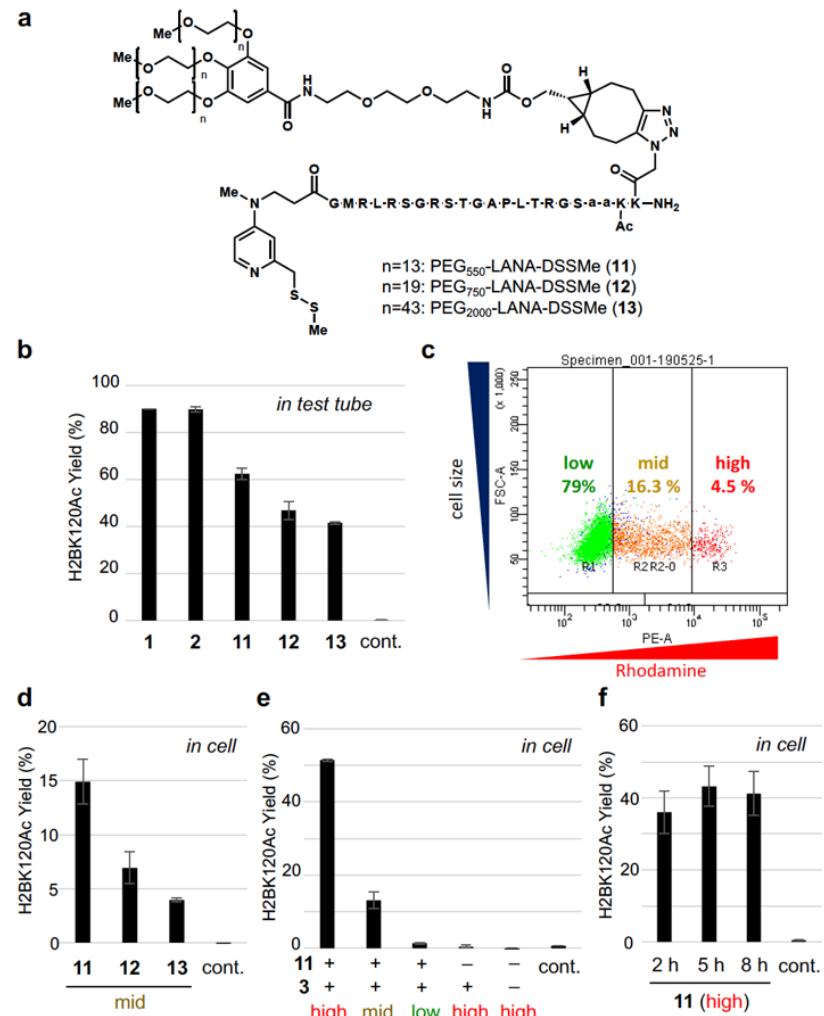

Figure 3. Histone H2BK 120 acetylation by PEG-LANA-DSH in living cells. (a) Structures of PEG-LANA-DSSMe (11-13). (b) LCMS/MS analysis of acetylation yield for H2BK120 on a recombinant nucleosome $(0.37 \mu \mathrm{M})$ treated with $3(10 \mathrm{mM})$ and TCEP $(0.1$ $\mathrm{mM})$ in the presence of $\mathbf{1}, \mathbf{2}$, or 11-13 $(2 \mu \mathrm{M})$ or in the absence of any catalysts (control) at $37^{\circ} \mathrm{C}$ for $5 \mathrm{~h}$. Error bars represent the data range of two independent experiments. (c) Representative dot plot of Rhodamine signals (Horizontal: PE-A) and cell size (Vertical: FSC-A). HeLa S3 cells were loaded with PEG-LANA-DSSMe and Dextran-Rhodamine, and analyzed by flow cytometer. Judging from the strength of Rhodamine signals, "high", "mid", and "low" fractions were defined and separated. (d-f) H2BK120 acetylation yield in living cells determined by LC-MS/MS analysis. HeLa S3 cells pretreated with BSO $(100 \mu \mathrm{M})$ were loaded with 11,12 , or 13 $(500 \mu \mathrm{M})$, TCEP $(2 \mathrm{mM})$, Dextran-Rhodamine $(0.5 \mathrm{mg} / \mathrm{mL})$, and/or $3(10 \mathrm{mM})$, and incubated with DMEM containing 3 (30 $\mathrm{mM}$ ) at $37^{\circ} \mathrm{C}$ for $8 \mathrm{~h}$ or indicated time. Acetylation yield of "high", "mid", or "low" fractions were shown. Control indicates acetylation yield without any treatment. Error bars represent the data range of two independent experiments.

For the cells containing a high level of $\mathbf{1 1}, \mathrm{H} 2 \mathrm{BK} 120$ was $51 \%$ acetylated (Figure $3 \mathrm{e}$ ). A time-course revealed that the conversion plateaued within $5 \mathrm{~h}$ (Figure $3 \mathrm{f}$ ). This reaction profile was consistent with the $1.2 \mathrm{~h}$ half-life of 7 in cells as determined from the time-lapse experiments (Figure $2 \mathrm{~d}$ ). In cells containing middle level of 11, 13\% H2BK120 was acetylated (Figure 3e), indicating that efficiency of the acetylation reaction was dependent on the catalyst concentration in cells.

Finally, to investigate the impact of the synthetically-introduced H2BK120 acetylation on the epigenome of living cells, we examined the level of H2BK120 ubiquitination (H2Bub), a mark regulating various chromatin functions, such as transcription and DNA damage response. ${ }^{14}$ We hypothesized that the acetyl group synthetically introduced at $\mathrm{H} 2 \mathrm{BK} 120$ by the chemical catalyst system would function as a protecting group, thus inhibiting 
the physiological ubiquitination reaction at the same residue mediated by ubiquitin ligases (Figure 4a). We compared the H2Bub level in cells containing high or low level of H2BK120 acetylation and found that $\mathrm{H} 2 \mathrm{Bub}$ level was significantly reduced in cells with high-level H2BK120 acetylation (Figure 4b). In contrast, the acetylation level at histone tail was not affected by high-level H2BK120 acetylation (Figure 4c). These data showcase that synthetic H2BK120 acetylation in living cells mediated by the chemical catalyst system can work as an inhibitor of $\mathrm{H} 2 \mathrm{Bub}$.

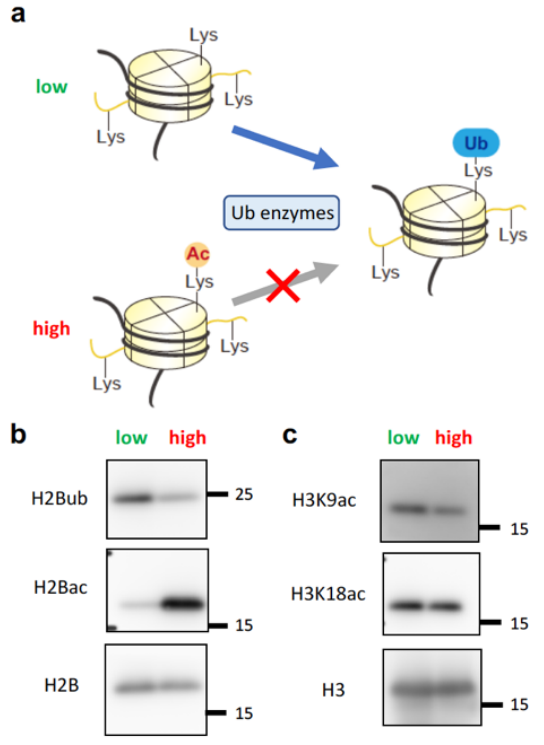

Figure 4. Synthetic histone acetylation by PEG-LANA-DSH 11 blocks H2B ubiquitination. (a) Schematics of possible effects of synthetic H2BK120 acetylation. (b), (c) Levels of H2BK120Ac (H2Bac), H2BK120Ub (H2Bub), and H3-tail acetylation (H3K9ac and $\mathrm{H} 3 \mathrm{~K} 18 \mathrm{ac}$ ) in $\mathrm{H} 2 \mathrm{BK} 120$-acetylated or non-acetylated cells. HeLa S3 cells pretreated with BSO $(100 \mu \mathrm{M})$ were loaded with 11 $(500 \mu \mathrm{M})$, TCEP $(2 \mathrm{mM})$, Dextran-Rhodamine $(0.5 \mathrm{mg} / \mathrm{mL})$, and 3 $(10 \mathrm{mM})$, incubated with DMEM containing $3(30 \mathrm{mM})$ at $37^{\circ} \mathrm{C}$ for $8 \mathrm{~h}$ for the chemical acetylation reaction and further incubated with DMEM in the absence of catalysts and donors at $37^{\circ} \mathrm{C}$ for 36 $\mathrm{h}$ for equilibrating the ubiquitination/de-ubiquitination processes mediated by the physiological enzymes in living cellular machinery. Histones were purified from "high" or "low" cell fraction obtained through sorting by flow cytometer, and analyzed by western blotting.

In summary, we developed a chemical system comprising a protease-resistant nucleosome-binding catalyst (PEG-LANA$\mathrm{DSH}$ ) and cell-permeable acetyl donor (NAC-Ac). This chemical catalyst system promoted regioselective lysine acetylation of chromatin histones in living cells for the first time. Our data indicate that increasing the size of PEG conferred the catalyst with greater in-cell stability, but reduced its acetylation activity, indicating that there is an optimum PEG length balancing the stability and catalyst activity. The optimized catalyst $\mathbf{1 1}$ efficiently promoted H2BK120 acetylation in living cells. We found that synthetic H2BK120 acetylation suppressed H2Bub. This mode of inhibitory action is conceptually analogous to regioselective protection of a functional group existing in a small organic molecule, a frequently-used strategy in organic synthesis. It is known that the presence of $\mathrm{H} 2 \mathrm{Bub}$ on nucleosomes can enhance the activity of DOT1L catalyzing H3K79 methylation, ${ }^{15}$ and both Dot1L and the H2B E3 ubiquitin ligase ring finger protein 20 (RNF20) are required for MLL-rearranged leukemia. ${ }^{16-17}$ Therefore, our catalyst system can be developed as a unique $\mathrm{H} 2 \mathrm{Bub}$ inhibitor, which may be applicable for anti- MLLrearranged leukemia.

\section{ASSOCIATED CONTENT}

\section{Supporting Information}

The Supporting Information is available free of charge on the ACS Publications website.

Synthetic protocols, experimental details and Figure S1-S4 (PDF)

\section{AUTHOR INFORMATION}

\section{Corresponding Author}

yamatsugu@mol.f.u-tokyo.ac.jp

skawashima@mol.f.u-tokyo.ac.jp

kanai@mol.f.u-tokyo.ac.jp

\section{Notes}

The authors declare no competing financial interests.

\section{ACKNOWLEDGMENT}

We thank to Dr. Kana Tanabe, Ms. Yuki Kobayashi, and Ms. Junko Kato for their assistance of experiments, Dr. Akiko Fujimura and Mr. Christopher W. Adamson for reading manuscript and helpful comments, and MAB Institute, Inc. for generating antiH2BK120Ac monoclonal antibody. This work was supported by MEXT/JSPS KAKENHI (JP17H01522/JP17K19479/JP20H00489 (M.K.), JP19KK0179 (M.K., K.Y., and S.A.K.), JP18H04536 (K.Y.), JP18H05534 (H.Ku.), JP17H01417 and JP18H05527 (H.Ki)), the Platform Project for Supporting Drug Discovery and Life Science Research (BINDS) from AMED (JP20am0101076 (H.Ku.)), JST ERATO (JPMJER1901 (H.Ku.)), the 'Epigenome Manipulation Project' of the All-RIKEN Projects supported by RIKEN.

\section{REFERENCES}

(1) Mirabella, A. C.; Foster, B. M.; Bartke, T., Chromatin deregulation in disease. Chromosoma 2016, 125, 75-93.

(2) Roadmap Epigenomics, C.; Kundaje, A.; Meuleman, W.; Ernst, J.; Bilenky, M.; Yen, A.; Heravi-Moussavi, A.; Kheradpour, P.; Zhang, Z.; Wang, J.; Ziller, M. J.; Amin, V.; Whitaker, J. W.; Schultz, M. D.; Ward, L. D.; Sarkar, A.; Quon, G.; Sandstrom, R. S.; Eaton, M. L.; Wu, Y. C.; Pfenning, A. R.; Wang, X.; Claussnitzer, M.; Liu, Y.; Coarfa, C.; Harris, R. A.; Shoresh, N.; Epstein, C. B.; Gjoneska, E.; Leung, D.; Xie, W.; Hawkins, R. D.; Lister, R.; Hong, C.; Gascard, P.; Mungall, A. J.; Moore, R.; Chuah, E.; Tam, A.; Canfield, T. K.; Hansen, R. S.; Kaul, R.; Sabo, P. J.; Bansal, M. S.; Carles, A.; Dixon, J. R.; Farh, K. H.; Feizi, S.; Karlic, R.; Kim, A. R.; Kulkarni, A.; Li, D.; Lowdon, R.; Elliott, G.; Mercer, T. R.; Neph, S. J.; Onuchic, V.; Polak, P.; Rajagopal, N.; Ray, P.; Sallari, R. C.; Siebenthall, K. T.; Sinnott-Armstrong, N. A.; Stevens, M.; Thurman, R. E.; Wu, J.; Zhang, B.; Zhou, X.; Beaudet, A. E.; Boyer, L. A.; De Jager, P. L.; Farnham, P. J.; Fisher, S. J.; Haussler, D.; Jones, S. J.; Li, W.; Marra, M. A.; McManus, M. T.; Sunyaev, S.; Thomson, J. A.; Tlsty, T. D.; Tsai, L. H.; Wang, W.; Waterland, R. A.; Zhang, M. Q.; Chadwick, L. H.; Bernstein, B. E.; Costello, J. F.; Ecker, J. R.; Hirst, M.; Meissner, A.; Milosavljevic, A.; Ren, B.; Stamatoyannopoulos, J. A.; Wang, T.; Kellis, M., Integrative analysis of 111 reference human epigenomes. Nature 2015, 518, 317-30.

(3) Hyun, K.; Jeon, J.; Park, K.; Kim, J., Writing, erasing and reading histone lysine methylations. Exp. Mol. Med. 2017, 49, e324.

(4) Eckschlager, T.; Plch, J.; Stiborova, M.; Hrabeta, J., Histone Deacetylase Inhibitors as Anticancer Drugs. Int. J. Mol. Sci. 2017, 18.

(5) Yamatsugu, K.; Kawashima, S. A.; Kanai, M., Leading approaches in synthetic epigenetics for novel therapeutic strategies. Curr. Opin. Chem. Biol. 2018, 46, 10-17.

(6) Muller, M. M.; Muir, T. W., Histones: At the Crossroads of Peptide and Protein Chemistry. Chem. Rev. 2015, 115, 2296-349.

(7) Chin, J. W., Expanding and reprogramming the genetic code of cells and animals. Annu. Rev. Biochem. 2014, 83, 379-408. 
(8) Yang, A.; Ha, S.; Ahn, J.; Kim, R.; Kim, S.; Lee, Y.; Kim, J.; Soll, D.; Lee, H. Y.; Park, H. S., A chemical biology route to site-specific authentic protein modifications. Science 2016, 354, 623-26.

(9) Wright, T. H.; Bower, B. J.; Chalker, J. M.; Bernardes, G. J. L.; Wiewiora, R.; Ng, W. L.; Raj, R.; Faulkner, S.; Vallee, M. R. J.; Phanumartwiwath, A.; Coleman, O. D.; Thezenas, M. L.; Khan, M.; Galan, S. R. G.; Lercher, L.; Schombs, M. W.; Gerstberger, S.; Palm-Espling, M. E.; Baldwin, A. J.; Kessler, B. M.; Claridge, T. D. W.; Mohammed, S.; Davis, B. G., Posttranslational mutagenesis: A chemical strategy for exploring protein side-chain diversity. Science 2016, 354

(10) Amamoto, Y.; Aoi, Y.; Nagashima, N.; Suto, H.; Yoshidome, D.; Arimura, Y.; Osakabe, A.; Kato, D.; Kurumizaka, H.; Kawashima, S. A.; Yamatsugu, K.; Kanai, M., Synthetic Posttranslational Modifications: Chemical Catalyst-Driven Regioselective Histone Acylation of Native Chromatin. J. Am. Chem. Soc. 2017, 139, 7568-76.

(11) Ishiguro, T.; Amamoto, Y.; Tanabe, K.; Liu, J.; Kajino, H.; Fujimura, A.; Aoi, Y.; Osakabe, A.; Horikoshi, N.; Kurumizaka, H.; Yamatsugu, K.; Kawashima, S. A.; Kanai, M., Synthetic Chromatin Acylation by an Artificial Catalyst System. Chem 2017, 2, 840-59.

(12) Hamajima, W.; Fujimura, A.; Fujiwara, Y.; Yamatsugu, K.; Kawashima, S. A.; Kanai, M., Site-Selective Synthetic Acylation of a Target
Protein in Living Cells Promoted by a Chemical Catalyst/Donor System. ACS Chem. Biol. 2019, 14, 1102-9.

(13) Harris, J. M.; Martin, N. E.; Modi, M., Pegylation: a novel process for modifying pharmacokinetics. Clin. Pharmacokinet. 2001, 40, 539-51.

(14) Meas, R.; Mao, P., Histone ubiquitylation and its roles in transcription and DNA damage response. DNA Repair (Amst) 2015, 36, 36-42.

(15) McGinty, R. K.; Kim, J.; Chatterjee, C.; Roeder, R. G.; Muir, T. W., Chemically ubiquitylated histone H2B stimulates hDot1L-mediated intranucleosomal methylation. Nature 2008, 453, 812-6.

(16) Daigle, S. R.; Olhava, E. J.; Therkelsen, C. A.; Majer, C. R.; Sneeringer, C. J.; Song, J.; Johnston, L. D.; Scott, M. P.; Smith, J. J.; Xiao, Y.; Jin, L.; Kuntz, K. W.; Chesworth, R.; Moyer, M. P.; Bernt, K. M.; Tseng, J. C.; Kung, A. L.; Armstrong, S. A.; Copeland, R. A.; Richon, V. M.; Pollock, R. M., Selective killing of mixed lineage leukemia cells by a potent smallmolecule DOT1L inhibitor. Cancer Cell 2011, 20, 53-65.

(17) Wang, E.; Kawaoka, S.; Yu, M.; Shi, J.; Ni, T.; Yang, W.; Zhu, J.; Roeder, R. G.; Vakoc, C. R., Histone H2B ubiquitin ligase RNF20 is required for MLL-rearranged leukemia. Proc. Natl. Acad. Sci. U. S. A. 2013 , $110,3901-6$ 

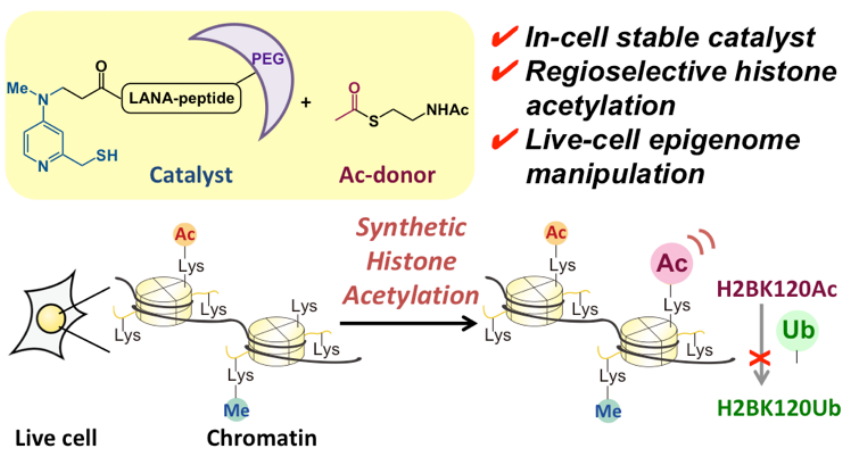